\title{
Assessment of patient delay in healthcare seeking behavior and associated factors among women with tuberculosis in governmental health in stitution, Mekelle City, Tigray, Ethiopia, 2012
}

\author{
Hiwot A. Hiluf ${ }^{1}$, Ashenafi D. Ayele ${ }^{2}$, Gereziher B. Abera ${ }^{2}$, Hailemariam B. Kahisay ${ }^{2}$, \\ Kalayou K. Berhe ${ }^{2}$ \\ ${ }^{1}$ Mekelle Hamlin Fistula Hospital Mekelle City, Tigray, North Ethiopia \\ ${ }^{2}$ Department of Nursing, College of Health sciences, Mekelle University, Tigray, North Ethiopia
}

\section{Email address:}

hiwotalemayo@yahoo.com(H. A. Hiluf), Ashenafidamte@yahoo.com (A. D. Ayele), gbamsc2002@gmail.com (G. B. Abera), aidhbk@gmail.com (H. B. Kahisay),kalushaibex@yahoo.com (K. K. Berhe)

\section{To cite this article:}

Hiwot A. Hiluf, Ashenafi D. Ayele, Gereziher B. Abera, Hailemariam B. Kahisay, Kalayou K. Berhe. Assessment of Patient Delay in Healthcare Seeking Behavior and Associated Factors among Women with Tuberculosis in Governmental Health in Stitution, Mekelle City, Tigray, Ethiopia, 2012. American Journal of Nursing Science. Vol. 3, No. 5, 2014, pp. 66-72. doi: 10.11648/j.ajns.20140305.12

\begin{abstract}
Objectives: was to assess patient delay in health care seeking behavior and associated factors among women with Tuberculosis in Mekelle City Governmental health facilities, Ethiopia 2012.Methods: The study design was Cross-sectional study and systematic random sampling technique was used to select participants from all governmental health facilities in Mekelle City. The study periods were from May 2012 to December, 2012.Data collection procedure was by using interviewer administered questionnaire. After completing data collection, data was coded and entered into SPSS version 20 software for analysis. univariate, Bivariate and multivariate logistic regression was computed and data was presented in texts, tables and figure. Result: A total of 257 participants were interviewed using standardized structured questionnaire and included in the analysis. Of those 15 respondents were excluded from the analysis for gross incompleteness and inconsistency of responses, made a response rate of $94.16 \%$. The mean age of respondents was30.59+12.61 years ranging from 16-75 years old. Being in age group $>55$ (AOR: $0.23,95 \%$ CI $0.006,0.03$ ) was at risk for delay. Marital status $79(75.2 \%$ ) was also found to be significantly associated with patient delay to seeking care and was found to be at great risk delay in seeking care (AOR: 0.002, $95 \%$ CI $0.001,0.32$ ) than that of other (divorce\& widowed). Educational status was also found to be significantly associated with patient delay, illiterate 63(98.4\%) by adjacently (AOR: 27, 95\% CI 1.30, 60.45) was at risk for patient delay. Conclusion and Recommendation In conclusion the finding of this result showed that from the total respondents the reason of patient delay for seeking care for more than two third of the respondents were lack of money, illiteracy and being married was significantly associated with patient delay Therefore, Delay in care seeking behavior among women with TB more specific and effective education and income improvement needs work on self-esteem development on care seeking behavior among women's with TB.
\end{abstract}

Keywords: Patient Delay, Health Care Seeking, Associated Factors

\section{Introduction}

\subsection{Background}

Ethiopia is one of the countries with high burden of TB .ranked $8^{\text {th }}$ in the world with an estimated incidence rate of tuberculosis 163 per 100,000 of the population. The case detection rate was only $32.7 \%$ in 2005 with the WHO target of $70 \%$ detection rate. The treatment success rate $79 \%$ in 2004 compared with the WHO target of $85 \%$ success rate. It is important to recognize that components of the DOTs strategy are, in fact, responses to behavioral challenges in TB control. Despite the effectiveness of the DOTs strategy, delays in seeking care among tuberculosis patients Sex inequalities can lead to poorer access to health care and delays to diagnosis of tuberculosis in women. In a 
population-based survey we assessed health-seeking behavior in adults with long-term cough. The prevalence of cough was $1 \%$ (213) and $2 \%$ (279) in men and women, respectively ${ }^{1}$

The annual incidence of smear positive pulmonary TB is still high. Despite the implementation of Directly Observed Treatment Short course strategy over the past two decades. Delay in TB diagnosis increases the risk of transmission and economic costs to patients and communities at large. The median patient delay at any public health facility is alarmingly prolonged with studies reporting from 2 to 4 months globally, tuberculosis kills approximately 500 people daily $98 \%$ of deaths occurring in developing world, affecting mostly young adults in their most productive years. In recent years, TB has been reported to be the leading killer among HIV-infected people with weakened immune systems; and that a quarter of a million TB deaths are HIV-associated, with most of them in Africa ${ }^{2}$.

The Direct Observed Treatment Short course is the main strategy in the control of tuberculosis despite the effectiveness of the DOTS strategy; delays in seeking care among women tuberculosis patients are common in a number of developing countries. It has been reported that a reasonable number of tuberculosis patients treated by traditional medical practitioners before they seek care at healthcare facilities. Delays in seeking care for TB have also been associated with stigma, in parts where the disease is considered as a 'dirty' disease, which mainly affects poor people ${ }^{3,4}$.

\subsection{Statements of the Problem}

Worldwide about two-thirds of all known people with tuberculosis are women. Whether this proportion indicates a true difference in frequency between the sexes or an undernotification of female cases is subject to speculation. Long's findings indicate that sex inequalities lead to poorer access to health care and delays in diagnosis and treatment of tuberculosis in women. Studies have identified several factors that account for patient delay and health care service delay. Numerous studies have found association between patient delay and knowledge, awareness, stigma, external constraints, and gender differences 5 .

Burden of tuberculosis on public health is staggering, and has become of growing concerns to stakeholders in recent times worldwide. In 1993, the World Health Organization declared a state of global emergency for TB. By 1995, the directly observed treatment short-course strategy was established to achieve TB control. Two of the key component of a good TB control programs are an early diagnosis and prompt institutions of effective treatment. This is especially important in patients with smear positive pulmonary tuberculosis in order to reduce the transmission time of mycobacterium TB in the communities. TB case detection is predicted on passive case finding using sputum smear microscopy. This is limited and has not been able to confront the growing burden and transmission of $\mathrm{TB}$ in most developing countries ${ }^{6}$.

Study conducted in Tigray showed that women took more health-care actions than men, but chose less qualified providers and reported lower health expenditure per visit. Delay before seeking hospital treatment was longer for women (41 days) than men ( 19 days; $\mathrm{p}=0.04)$, and more men $(27 ; 36 \%)$ than women $(14 ; 14 \% ; p=0 \cdot 0006)$ reported giving a sputum sample at hospital. Sex-sensitive strategies for tuberculosis control are needed and should take into account sex differences in health-care seeking behavior as well as a possible sex bias among health-care providers ${ }^{7,8}$.

Little is known about delay in health care seeking behaviors among women TB patients towards tuberculosis. Thus, this study attempts to assess the patient delay in health care seeking behavior and associated factors among women with TB.

\subsection{Justification of the Study}

Despite the fact that similar studies are undertaken about delays in treatment and care seeking behavior in Ethiopia and elsewhere in the world, factors influencing this delay varies from culture to culture and from local to local. Identifying factors contributing to delay in treatment among women TB patients have paramount importance to reduce morbidity and could contribute in addressing the behaviors and challenges particular to the area under study. There are few studies done on delay and care seeking behaviors among TB patients. This study was assessed patient delay of care seeking. The aim of this study is to generate information which contributes for action regarding patient delay in health care seeking and in Mekelle Governmental health facilities. The finding of this study will be help full for further study by researchers and for service provision by health facilities and health policy makers. Conducting this study will be help full in reducing risk of transmission of tuberculosis in the community.

\section{Methodology}

\subsection{Study Area and Period}

Mekelle is found in Tigray region, northern Ethiopia $778 \mathrm{Km}$ far from Addis Ababa. The city has an area of 28 squares Km, which comprises 7 KifleKetemas and 107 Ketena. The ketenas are the smallest administrative unites of the city .where as kifleketenas are the middles administer. According to 2011 population and housing censes of Ethiopia, the total population of the city was 264,907 among those total women's of reproductive age in the city was 57,310.Among those age above fifteen was first quarter reported 290 women infected with TB. And Tuberculosis infected individual is high in the city and report of Mekelle regional health bureau showed that prevalence rate in 2003 E.C (2011) is 168 per 100,000 populations which are higher than the country's prevalence rate. Because of this TB is a serious public health concern in the city ${ }^{20}$. The study area was conducted in Mekelle Governmental health facility. Mekelle has four public hospitals, four private hospitals, and 12 health centers, and 38 private clinics. This study was 
conducted from May to December 2012.

\subsection{Study Design, Population and Eligibility Criteria}

Study design was institution based cross sectional study. Source population was all TB diagnosed women patients in the selected governmental health facilities in Mekelle city during the study period. Study population was all selected women with TB from the selected health facilities in 3 hospitals and 9 health centers which is TB attending in Mekelle city. All above 15 years old TB diagnosed women in Mekelle public health facility were included in this study. Mentally and seriously ill women were excluded from this study.

\subsection{Sample Size Determination}

This study was used single mean population proportion formula, and with the assumption of $95 \%$ confidence interval and a precision of $5 \%$. To compensate for some nonresponse $10 \%$ wasadded, since no study separately among women with TB, 50\% prevalence was taken., then the initial sample size was $n=384$ Since my study population is less than 10,000 by using correction formula. Finally By adding $10 \%$ non-response rate the final sample size was 257 . So to have equaled number of women with TB from the nine governmental health facilities sample size of each public facility allocated based on proportion to size allocation formula according to patient flow.

\subsection{Sample Procedure}

Systematic random sampling was used to select the study participants. There are twelve Governmental health facilities in Mekelle city, so data was collected from all Governmental health facilities. The study participants were allocated from each health facilities by using proportion allocation to size.

\subsection{Data Collection Procedure and Data Tool}

The Interviewer questionnaire developed from delay care seeking behavior with TB which was the study conducted in Ethiopia. The questionnaire includes socio-demographic variables, health care seeking and delay variables. The questionnaire was translated in to local language Tigrigna before it reaches to the respondents. Data was collected by six diploma graduated nurses with previous data collection experience. The principal investigator and supervisor were check for completed of each collected data daily

\subsection{Data Quality Assurance, Study Variables and Operational Definition}

Those data collectors were trained for three days on data collection process in general before they start data collection. $5 \%$ of the questionnaire was pretested at Wukro town for individuals with same inclusion criteria. Necessary correction was made based on the pretested result to avoid any confusion and for better completed of the questions. Dependent variable Patient delay of Care seeking and
Independent variables were Age, Marital status, Religions, Ethnicity, Occupation, Education, Monthly income. Operational definitions: Patient delay-Is the time from onset of first symptom to visit health facility above two week, Health seeking behavior-To those entire things humans do to prevent diseases and to detected diseases in symptomatic stage.

\subsection{Data Analysis}

After data collection completed, data were coded during data entry and entered in to SPSS version 20 software for analysis.Univariate analysis was done on socio-demographic characteristics to see the frequency of each variable. Bivariate analysis was used to test association between dependent variable and each independent variable. Significant association between dependent and independent variables was interpreted accordingly.

Multivariate analysis was employed to control for possible the effects of the two or more independent variables on the dependent variables.

\subsection{Ethical Clearance}

This study was conducted after the approval of the proposal by Mekelle University, college of health sciences ethical review committee. Permission was also obtained from Mekelle zonal health office and respective public health facilities. The purpose of this study was explained to selected patients and Informed consent was obtained from patients who were willing to participate in this study. Confidentiality was kept by omitting participant's personal identification.

\section{Results}

\subsection{Socio-Demographic Characteristics of Study Participants}

A total of 257 participants were interviewed using standardized structured questionnaire and included in the analysis. Of those 15 respondents were excluded from the analysis for gross incompleteness and inconsistency of responses, made a response rate of $94.16 \%$. The mean age of respondents was 30.59+12.61 years ranging from 16-75 years old. Majority of the respondents were in the age group 35-44 which is 91(37.6\%).Most women TB patients $105(43.4 \%)$ were married, while $87(36 \%)$ were single, $32(13.2 \%), \quad 18(7.4 \%)$ were divorced and widowed respectively. Sixty-four participants (26.4\%), 37(15.3\%) were illiterate and literate basic respectively. While $67(27.7 \%)$ TB patients attended high school and 11(4.5\%) participants were bachelor degree holders. The distribution of religious background in the TB patients were orthodox 207(85.5\%), Muslims 9(3.7\%) and protestants 26 (10.7\%).Majority of respondents $208(90.1 \%)$ were from urban residence. The employment status of TB patient reflects 58(24\%) of TB patients were Jobless, While house wife TB patients, privately and government employed accounted for $58(24.0 \%), 43(17.8 \%)$ and $29(12.0 \%)$ of $\mathrm{TB}$ 
patients respectively, only $17(7.0 \%)$ of TB patients were merchants (Table 1).

\subsection{Health Care Seeking Behavior of Women with TB}

From the total respondents $13.6 \%, 23.1 \%, 20.2 \%, 43 \%$ started to recognize sign and symptoms like cough, fever, night sweating and weight loss $1,2,3$ and $>3$ weeks before they came to health institution respectively of the frequency percent. The majority of patients 177 (73.1\%) made their first consultation at health in situations. A total of 59 $(24.38 \%)$ had not sought informal care for their symptoms between the onset of their illness and their first consultation at a public health facility, from whom $45(18.6 \%)$ had been treated with holy water, $6(2.5 \%)$ by religious/traditional healers, $5(2.1 \%)$ by ten quay/kalicha and $9(3.7 \%)$ others (Table 2).

Table 1. Socio-demographic characteristics of women with TB $(N=242)$ in Mekelle governmental health facilities Ethiopia, May-Dec 2012.

\begin{tabular}{|c|c|c|}
\hline Variables & Frequency & Percent (\%) \\
\hline \multicolumn{3}{|l|}{ Age } \\
\hline $15-24$ & 36 & 14.9 \\
\hline $25-34$ & 50 & 20.7 \\
\hline $35-44$ & 91 & 37.6 \\
\hline $45-54$ & 29 & 12.0 \\
\hline$>55$ & 36 & 14.9 \\
\hline \multicolumn{3}{|l|}{ Religion } \\
\hline Orthodox & 207 & 85.5 \\
\hline Muslim & 9 & 3.7 \\
\hline Protestant & 26 & 10.7 \\
\hline \multicolumn{3}{|l|}{ Residence } \\
\hline Rural & 218 & 90.1 \\
\hline Urban & 24 & 9.9 \\
\hline \multicolumn{3}{|l|}{ Ethnicity } \\
\hline Amhara & 17 & 7.0 \\
\hline Afar & 1 & 0.4 \\
\hline Tigre & 223 & 92.1 \\
\hline \multicolumn{3}{|l|}{ Marital status } \\
\hline Single & 87 & 36.0 \\
\hline Separated/divorced & 32 & 13.2 \\
\hline Married & 105 & 43.4 \\
\hline Widowed & 18 & 7.4 \\
\hline \multicolumn{3}{|l|}{ Education background } \\
\hline Illiterate & 64 & 26.4 \\
\hline Literate basic & 37 & 15.3 \\
\hline $1-8$ & 43 & 17.8 \\
\hline $9-12$ & 67 & 27.7 \\
\hline Diploma & 20 & 8.3 \\
\hline Bachelor & 11 & 4.5 \\
\hline \multicolumn{3}{|l|}{ Occupation status } \\
\hline Employed by government & 29 & 12.0 \\
\hline Employed by private & 43 & 17.8 \\
\hline House wife & 58 & 24 \\
\hline Merchant & 17 & 7 \\
\hline Student & 28 & 11.6 \\
\hline Others & 9 & 3.7 \\
\hline \multicolumn{3}{|l|}{ Income } \\
\hline$<200$ & 51 & 21.1 \\
\hline $200-400$ & 47 & 19.4 \\
\hline $401-600$ & 67 & 27.7 \\
\hline $601-1000$ & 66 & 27.3 \\
\hline$>1000$ & 11 & 4.5 \\
\hline
\end{tabular}

Table 2. Health care seeking among pulmonary tuberculosis patients $(n=242)$ in Mekelle governmental health facilities May-Dec 2012

\begin{tabular}{lll}
\hline Sources of alternative treatment & Frequency & Percent \\
\hline Holy water & 45 & 18.6 \\
Religious healers & 6 & 2.5 \\
Sheke/Kalicha & 5 & 2.1 \\
Health institution & 177 & 73.1 \\
Others* & 9 & 3.7 \\
\hline
\end{tabular}

\subsection{Patient Delay in Health Care Seeking}

\subsubsection{Socio-Demographic Variables and Patient Delay}

The overall prevalence of Patient delay in health care seeking behavior was $75.6 \%$.Respondents categorized in age group 35-44 which is $61(33.5 \%)$ had longest delay to visit health institution than the other age group categories. Respondents who are illiterate were more likely to delay longer in first seeking care at a health facility which is 63(34.4\%).Among women with TB patient respondents' house wife and Jobless had longest delay to visit health institution which is $43(23.5 \%), 47(25.7 \%)$ respectively. Respondents residing urban area had longer delay to visit health institution than respondents residing rural area which was $160(87.4 \%)$. Regarding marital status $79(43.2 \%)$ married respondents were more likely to delay in visiting to health institution. Prevalence of patient delay was higher among respondents who perceived their illness as not serious which is $61(33.33 \%)$.(Table 3$)$

\subsubsection{Reason for Patient Delay in Health Care Seeking}

Common reasons given for delay were: Perception of illness as not serious $71(29.3 \%)$, Lack of information $58(24 \%)$, Lack of money $55(22.7 \%)$ and other problems 39 (16.1\%) (Fig 1).

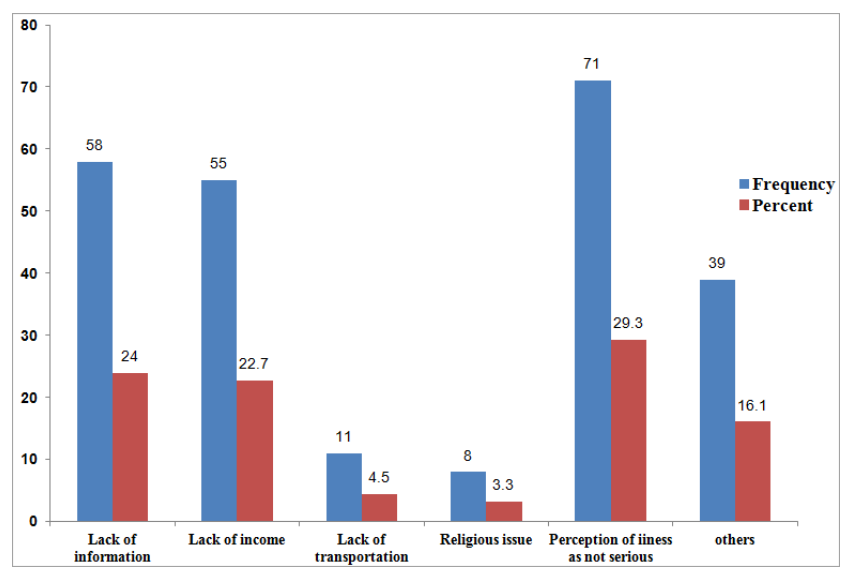

Fig 1. Reason for patient delay in health care seeking among women with $T B N=242$

\subsection{Factors Associated with Patient Delay in Healthcare Seeking}

\subsubsection{The Association between Delay in Seeking Care and Socio Demographic Variables}

The association between socio-demographic variables and delay in health seeking care was tested in a binary logistic 
regression both bi-variants and multi-variants analysis was done and presented in. Among socio-demographic variables, age, marital status educational status, and income were significantly associated with delay in seeking care among woman with TB treatment. Accordingly age group 3544were found to be 61(33.3\%) significantly associated with patient delay. Being in age group $>55$ (AOR: $0.23,95 \% \mathrm{CI}$ $0.006,0.03)$ was at risk for delay in TB diagnosis $(p=0.012)$.Marital status was also found to be Patient who already married $79(75.2 \%)$ significantly associated with patient delay and (AOR: $0.002,95 \%$ CI $0.001,0.32$ ) was found to be at risk patient delay $(\mathrm{p}=0.006)$ were four times delay than that of other (divorce, widowed).And also who were no married (single) found associated with delay (AOR: $0.02,95 \%$ CI 0.001 .0 .41$)(\mathrm{p}=0.011)$.

Educational status was also found to be associated patient delay $\quad(p=0.035) \quad$ accordingly illiterate $63(98.4 \%)$ significantly associated with patient delay by adjacently (AOR: 27, 95\% CI 1.30, 60.45) was at risk for patient delay. Regarding respondents income it was also found to be significantly associated with patient delay. Almost half of the respondents have monthly income of less than 200.00 ETB (AOR: 53.249 95\% CI 2.8, 1. 12) was significantly associated with patient delay $(\mathrm{p}=.008)$. (Table 3$)$

Table 3. Factors associated with delay in health seeking care

\begin{tabular}{|c|c|c|c|c|}
\hline \multirow{2}{*}{ Socio demographic variables. } & \multicolumn{4}{|c|}{ Delay in health Care seeking behavior among woman with $\mathrm{TB}: \mathrm{n}=242$} \\
\hline & 2 wks & $>2$ wks & COR 95\% CI & AOR 95\% CI \\
\hline \multicolumn{5}{|l|}{ Age } \\
\hline $15-24$ & $5(13.9 \%)$ & $31(86.1 \%)$ & 1 & 1 \\
\hline $25-34$ & $9(18.0 \%)$ & $41(82.0 \%)$ & $0.74(0.23,2.45)$ & $1(0.2,4.98)$ \\
\hline $35-44$ & $30(33.0 \%)$ & $61(67.0 \%)$ & $0.32(0.12,0.43)$ & $0.34(0.08,1.49)$ \\
\hline $45-54$ & $9(31.0 \%)$ & $20(69.0 \%)$ & $0.36(0.11,1.23)$ & $0.27(0.03,2.22)$ \\
\hline$\geq 55$ & $9(16.7 \%)$ & $30(83.3 \%)$ & $0.81(0.22,2.93)$ & $0.06(0.01,0.53)^{*}$ \\
\hline Widowed & $1(5.6 \%)$ & $17(94.4 \%)$ & 1 & 1 \\
\hline Single & $28(32.2 \%)$ & $59(67.8 \%)$ & $0.013(0.02,0.98)$ & $0.02(0.001,0.41)^{*}$ \\
\hline Married & $26(24.8 \%)$ & $79(75.2 \%)$ & $0.18(0.02,1.41)$ & $0.02(0.000,0.32)^{*}$ \\
\hline Divorces/separate & $4(12.5 \%)$ & $28(87.5 \%)$ & $0.41(0.40,3.9)$ & $0.09(0.004,2.06)$ \\
\hline \multicolumn{5}{|l|}{ Educational Status } \\
\hline Bachelor & $2(18.2 \%)$ & $9(81.8 \%)$ & 1 & 1 \\
\hline Illiterate & $1(1.6 \%)$ & $63(98.4 \%)$ & $41(1.15,170.56)$ & $27(1.30,604.45)^{*}$ \\
\hline $9-12$ & $22(32.8 \%)$ & $46(67.2 \%)$ & $0.45(0.09,2.28)$ & $0.39(0.005,3.13)$ \\
\hline Diploma & $14(70.8 \%)$ & $29(30.0 \%)$ & $0.09(0.02,0.60)$ & $0.02(0.002,0.02$ \\
\hline \multicolumn{5}{|l|}{ Income } \\
\hline$<200$ birr & $1(2.0 \%)$ & $50(98.0 \%)$ & $18.75(1.73,203.21)$ & $53.249(2.8,1.12)^{*}$ \\
\hline $200-400$ & $26(55.3 \%)$ & $21(44.7 \%)$ & $0.3(0.07,1.29)$ & $0.26(0.03,1.96)$ \\
\hline $401-600$ & $13(19.4 \%)$ & $54(81.6 \%)$ & $1.56(0.36,6.70)$ & $4.65(0.63,34.21)$ \\
\hline $601-1000$ & $16(24.2 \%)$ & $50(75.8 \%)$ & $3.125(0.042,3.207)$ & $3.5(0.45,26.90)$ \\
\hline$>1000$ & $3(27.3 \%)$ & $8(72.7 \%)$ & 1 & 1 \\
\hline
\end{tabular}

The association between other selected variables were tested in a binary logistic regression both Bivariate and multi-variant analysis was also done and as result a place where they seek care and reason to delay was found to be significantly associated with patient delay. Accordingly from the total respondents the reason of patient delay more than two third of reported lack of information 45(77.6\%). And that patient who reported perception of illness as not serious on TB service had twice more at risk than other causes for reason for patient delay. Thirty (71.4\%) of respondent reported that their reason for patient delay was lack of money and it had also significantly associated with patient delay. Accordingly those respondents who reported lack of money to get TB treatment (AOS $=0.23,95 \%$, CI0.006, 0.03) more at risk than other cause of reason for delay. $(\mathrm{p}=0.039)($ Table 4$)$

Table 4. Selected variables evaluated for possible association with length of care seeking behavior among women with TB, Mekelle City,2012 (n=242).

\begin{tabular}{|c|c|c|c|c|}
\hline \multirow{3}{*}{ Reason for Delay } & \multicolumn{4}{|c|}{ Delay in Health Care seeking behavior } \\
\hline & \multicolumn{2}{|c|}{ Time of seeking } & \multirow{2}{*}{ COR 95\% CI } & \multirow{2}{*}{ AOR 95\% CI } \\
\hline & 2 wks & $>2$ wks & & \\
\hline \multicolumn{5}{|c|}{ Where you go to seek care } \\
\hline Holly water & $6(13.3 \%)$ & $39(86.7 \%)$ & $8.13(1.7,39.08)$ & $11.6(0.9,153.65)$ \\
\hline Religious healer & $1(16.7 \%)$ & $5(83.3 \%)$ & $6.25(0.5,77.5)$ & $2.1(0.01,54.0)$ \\
\hline wizare, "Kalicha" & $1(20.0 \%)$ & $4(80.0 \%)$ & $5(0.4,64.40)$ & $0.81(0.004,16.00)$ \\
\hline Health institution & $46(26.0 \%)$ & $131(74.0 \%)$ & $3.63(0.92,13.85)$ & $0.210(0.000,1.5174)$ \\
\hline Others & $5(55.6 \%)$ & $4(44.6 \%)$ & 1 & \\
\hline \multicolumn{5}{|l|}{ Reason to delay } \\
\hline Lack of information & $13(22.4 \%)$ & $45(77.6 \%)$ & $1.04(0.395,2.732)$ & $0.50(0.10,2.26)$ \\
\hline Lack of money & $12(28.6 \%)$ & $30(71.4 \%)$ & $0.4(0.14,0.89)$ & $0.23(0.006,0.03)^{*}$ \\
\hline Lack of transportation & $1(9.1 \%)$ & $10(90.9 \%)$ & $3(0.337,26.710)$ & $1.51(0.04,62.06)$ \\
\hline
\end{tabular}




\begin{tabular}{|c|c|c|c|c|}
\hline \multirow{3}{*}{ Reason for Delay } & \multicolumn{4}{|c|}{ Delay in Health Care seeking behavior } \\
\hline & \multicolumn{2}{|c|}{ Time of seeking } & \multirow{2}{*}{ COR 95\% CI } & \multirow{2}{*}{ AOR 95\% CI } \\
\hline & 2 wks & $>2$ wks & & \\
\hline Religious issue & $1(12.5 \%)$ & $7(87.5 \%)$ & $2.1(0.23,19.40)$ & $0.7(0.03,14)$ \\
\hline Perception of illness as not serious & $23(27.4 \%)$ & $61(72.6 \%)$ & $1.83(0.70,4.94)$ & $2.56(0.6,10.96)$ \\
\hline $\begin{array}{l}\text { Others } \\
\text { protect oneself from getting TB }\end{array}$ & $9(23.1 \%)$ & $30(76.9 \%)$ & 1 & 1 \\
\hline Yes & $48(29.3 \%)$ & $116(70.7 \%)$ & 1 & 1 \\
\hline do not know & $8(14.5 \%)$ & $47(85.5 \%)$ & $1.977(2.243,4.284)$ & $2.923(0.215,4.033)$ \\
\hline \multicolumn{5}{|c|}{ Eating good quality food and maintain hygienic care } \\
\hline Yes & $48(30.0 \%)$ & $112(70.0 \%)$ & 1 & 1 \\
\hline No & $11(13.4 \%)$ & $71(86.6 \%)$ & $0.037(0.251,0.959)$ & $0.257(0.009,3.471)$ \\
\hline \multicolumn{5}{|l|}{ Having /Living in clean environment } \\
\hline Yes & $45(28.7 \%)$ & $112(71.3 \%)$ & $0.037(0.251,0.959)$ & $0.545(0.345,7.528)$ \\
\hline No & $14(16.5 \%)$ & $71(83.5 \%)$ & 1 & 1 \\
\hline Yes & $45(29.6 \%)$ & $107(70.4 \%)$ & 1 & 1 \\
\hline No & $14(15.6 \%)$ & $76(84.4 \%)$ & $0.015(0.225,0.854)$ & $0.620(0.186,2.724)$ \\
\hline \multicolumn{5}{|c|}{ Abiding ( Faithful, keep) by doctors advice } \\
\hline Yes & $44(28.6 \%$ & $110(71.4 \%)$ & 1 & 1 \\
\hline No & $15(17.0 \%)$ & $73(83.0 \%)$ & $0.047(0.266,0.990)$ & $0.693(0.357,4.704)$ \\
\hline
\end{tabular}

*significant at $\mathrm{p}<0.05$

\section{Discussion}

This study has identified the delay care seeking behaviors from onset of symptoms the total was above two weeks of the patients delay among women's with TB. In Southern Nigeria revealed that Women were found to have a significantly longer total delay before diagnosis of tuberculosis 3.3 months for women. Factors associated patient delay in care seeking behavior among woman with TB early detection needs such efforts therefore need to focus on women among who are illiterate ensuring that educational materials are adapted to their level of education and literacy and are culturally appropriate developed. So respondents categorized in age group 35-44(33.3\%) had longest delay to visit health institution than the other age group categories and who are illiterate were more likely to delay longer in first seeking care at a health facility which is 63(34.4\%).Similar study in Amazon(2008) female gender was associated with a $52 \%$ longer delay; and education less than complete secondary schooling was associated with a $44 \%$ longer delay and first consultation $(>21$ days since onset of illness) was significantly higher among patients with no formal education (Adjusted Odds Ratio $(\mathrm{AOR})=2.46$; 95\%Confidence Interval (CI) $=1.21-5.01$ ), among those treated first by a private and/or traditional practitioner $(\mathrm{AOR}=2.9 ; 95 \% \mathrm{CI}=1.42-6.08)^{9,12}$.

Educational status was also found to be associated patient delay. Accordingly being illiterate AOR: $27,95 \%$ CI 1.30 , 60.45 ) was at risk for patient delay. Consequently $55(22.7 \%)$ of respondent reported that their reason for patient delay was lack of money accordingly those respondents who reported lack of money to get TB treatment. Similarly those who reported lack of money to get TB treatment and care service had 3 times more at risk than other causes for reason for patient delay. When they visited traditional healers, women had a significantly longer delay first visit to health care providers to diagnosis 3.0 months for women). More women $(35 \%)$ visited traditional healers before diagnosis, and were more likely to receive more complicated charms from traditional healer most of them. Tended to visit the government medical establishment first if they knew that free TB treatment was available, but women did not and that patient who reported lack of information on TB service had twice more at risk than other causes for reason for patient delay. ${ }^{13}$

Accordingly the longest patient delay (median $=90$ days) recorded was among patients who had been treated with holy water. Patients who have been treated with holy water and by private practitioners contributed $52 \%$ and $27 \%$ of the total delayed consultations respectively also Patient delay was longer than 90 days in $15 \%$ of cases delay among women was $54 \%$; $95 \%$ CI $(54 \%, 58 \%)$. $(3,4)$ Hence the majority of patients $177(73.1 \%)$ made their first consultation at health institutions total of 59 patients $(24.38 \%)$ had not sought informal care for their symptoms between the onset of their illness and their first consultation at a public health facility, from whom $45(18.6 \%)$ had been treated with holy water, 6 $(2.5 \%)$ by religious/traditional healers, Five $(2.1 \%)$ by Sheke/kalicha and 9 (3.7\%) and others (Table 2).Likewise study conducted in Tigray region revealed that the median delay in care seeking behavior was likely to have the risk of morbidity, mortality and the disease transmission to contracts (7, 18).Understanding delayed behavior in care seeking among woman with TBcase finding health it helps promotion special know how on TB prevention. Marital status was also found to be Patient who already married 79(75.2\%) significantly associated with patient delay and (AOR: 0.002, $95 \%$ CI $0.001,0.32$ ) was found to be at risk patient delay $(\mathrm{p}=0.006)$ delay than that of other (divorce\& widowed).Generally woman with lower levels education, income and other such time in gives priority for themselves, also living and working in crowding, poor ventilations 
leading to it might be the disease become worsens.

\section{Conclusion and Recommendations}

This study assessed patient delay in health care seeking behavior among women with $\mathrm{TB}$ and identifies factors associated. Accordingly from the total respondents the reason of patient delay for more than two third of the respondents were lack of money, illiteracy and being married was significantly associated with patient delay. Based on this finding we recommend the following activates to be accomplished: Regional Health Bureau should participate in preparing community based awareness creation programs towards women with $\mathrm{TB}$, as a result it may have its own impact in improving self esteem and behavioral change of women to early seek for TB treatment.

\section{Acknowledgements}

Our deepest gratitude goes to Mekelle University, college of health sciences, department of Nursing for every support we received to do this research. And we would like to extend our sincere gratitude to the supervisors and the study participants for being involved in the study.

\section{References}

[1] World Health Organization: Global tuberculosis Control: epidemiology, strategy, Financing Geneva, Switzerland: WHO report (2009).

[2] Berhane $\mathrm{Y}$ etal .Patient and health service delay in the diagnosis of pulmonary tuberculosis in Ethiopia. BMC Public Health2002, 2(1):23.

[3] Ministry of Health Uganda: Guidelines for National demonstration and Training Districts implementing DOTS, Ministry of Health NTLP Working draft Kampala World Health Organization. Global tuberculosis control: WHO Report (2003).

[4] Gender difference in treatment seeking behaviors' of tuberculosis cases in rural communities of Bangladesh Southeast Asian Journal of Tropical Medicine and Public Health 35, 126-135.

[5] Bulla A. [Global review of tuberculosis morbidity and mortality in the world (1961-1971)]. World Health Stat Rep1977; 30(1):2-38.

[6] Okanurak, K., etalDeterminants of patient delay in seeking treatment among pulmonary tuberculosis cases in a government specialist hospital in Ibadan, Nigeria (2004)

[7] MESIFN M, etal. Delay and care seeking behavioral among Tuberculosis patient in Tigray of northern Ethiopia J health .DEV (.2005).
[8] Factors influencing care seeking behavior of chest symptomatic: a community based survey involving rural and urban population in Tamil Nadu, South India. Tropical Medicine and International Health 2003, 8 (4):336-341.

[9] J.C., Onyeneho, N.G, etal. Where do tuberculosis patients go for treatment before reporting to DOTS clinics southern Nigeria. Tanzania Health Research Bulletin 9, 94-101. . (2007).

[10] Elizabeth 1 Corbett, Barbara Marston, Gavin J Churchyard, Kevin M De Cock. Tuberculosis in sub- Saharan Africa: opportunities, challenges, and change in the eraof antiretroviral treatment. Lancet 2006; 367:926-958.

[11] G. Chenget al. Factors affecting delays in tuberculosis diagnosis in rural China 2004

[12] Carolyn M. Fordet al factors Associated with Delayed Tuberculosis Test-seeking Behavior in the Peruvian Amazon 2008

[13] Onyeneho, N.G, et al. Where do tuberculosis patients go for treatment before reporting toDOTS clinics southern Nigeria. Tanzania Health Research Bulletin 9, 94-101. . (2007).

[14] Pronyk RM et al Assessing health seeking behavior among tuberculosis patients in rural South Africa 2003

[15] Assessing health seeking behaviour among tuberculosis patients in rural South Africa.Pronyk RM, Makhubele MB,Hargreaves JR,Tollman SM, Hausler HP. Department of Community Health, University of the Wiltwatersrand, Medical School, Parktown, Johannesburg, South Africa. pronyk@soft.co.za

[16] Factors Associated with Delayed Tuberculosis Test-seeking Behavior in the Peruvian Amazon Carolyn M. Ford , $\uparrow$ Angela M. Bayer , $\dagger$ Robert H. Gilman , DamiOnifade , Colleen Acosta, Lilia Cabrera ,Carlos Vidal, and Carlton A. Evans

[17] Community knowledge, attitudes and practices towards tuberculosis and its treatment in Mpwapwa District, central Tanzania. Tanzania Health Research Bulletin 9, 3843.Okeibunor Lorenz, $\mathrm{N}$.at .el Health in the Urban Environment: Experience from Dar salaam, Tanzania. Annals of the New York Academy of Sciences 1023, 159-163. (2004)

[18] Mengiste $\mathrm{M}$ et al Delayed consultation among pulmonary tuberculosis patients: a cross sectional study of 10 DOTS districts of Ethiopia (2006).

[19] Mengiste M Mesfin et al Delays and care seeking behavior among tuberculosis patients in Tigray of northern Ethiopia (2002).

[20] Regional health bureau population census of housing, Mekelle, Tigray, Ethiopia.(2011).

[21] Patient delay in pure sat province Cambodia. (2009).

[22] Assessment of acceptability provider patient among TB on DOTS in Adama city Addis Ababa Ethiopia (.2008). 